\title{
TRUNCATED TOEPLITZ OPERATORS ON FINITE DIMENSIONAL SPACES
}

\author{
Joseph A. Cima, William T. Ross and Warren R. Wogen
}

Abstract. In this paper, we study the matrix representations of compressions of Toeplitz operators to the finite dimensional model spaces $H^{2} \ominus B H^{2}$, where $B$ is a finite Blaschke product. In particular, we determine necessary and sufficient conditions - in terms of the matrix representation - of when a linear transformation on $H^{2} \ominus B H^{2}$ is the compression of a Toeplitz operator. This result complements a related result of Sarason [6].

Mathematics subject classification (2000): Toeplitz operators, model spaces, Clark operators, matrix representations.

Key words and phrases: 47B35, 47B32, 30D55.

\section{REFERENCES}

[1] J. A. Cima, A. L. Matheson And W. T. Ross, The Cauchy transform, Mathematical Surveys and Monographs, vol. 125, American Mathematical Society, Providence, RI, 2006.

[2] D. N. CLARK, One dimensional perturbations of restricted shifts, J. Analyse Math. 25 (1972), 169-191.

[3] P. L. DuREN, Theory of $H^{p}$ spaces, Academic Press, New York, 1970.

[4] S. R. GARCIA AND M. PUTINAR, Complex symmetric operators and applications, Trans. Amer. Math. Soc. 358 (2006), no. 3, 1285-1315 (electronic).

[5] D. SARASON, Sub-Hardy Hilbert spaces in the unit disk, University of Arkansas Lecture Notes in the Mathematical Sciences, 10, John Wiley \& Sons Inc., New York, 1994.

[6] D. SARASON, Algebraic properties of truncated Toeplitz operators, Operators and Matrices 1 (2007), no. $4,491-526$ 